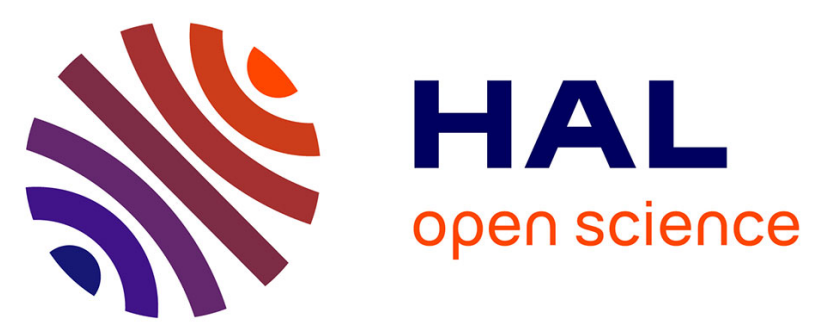

\title{
Time-varying stabilizing feedback for a class of nonlinear discrete-time control systems
}

Mohamed Bensoubaya, Abdelhak Ferfera, Abderrahman Iggidr

\section{To cite this version:}

Mohamed Bensoubaya, Abdelhak Ferfera, Abderrahman Iggidr. Time-varying stabilizing feedback for a class of nonlinear discrete-time control systems. 34th IEEE Conference on Decision and Control, Dec 1995, New Orleans, Louisiana, United States. pp.512-514, 10.1109/cdc.1995.478943 . hal-01862878

\section{HAL Id: hal-01862878 \\ https://hal.inria.fr/hal-01862878}

Submitted on 30 Aug 2018

HAL is a multi-disciplinary open access archive for the deposit and dissemination of scientific research documents, whether they are published or not. The documents may come from teaching and research institutions in France or abroad, or from public or private research centers.
L'archive ouverte pluridisciplinaire HAL, est destinée au dépôt et à la diffusion de documents scientifiques de niveau recherche, publiés ou non, émanant des établissements d'enseignement et de recherche français ou étrangers, des laboratoires publics ou privés. 


\title{
TIME-VARYING STABILIZING FEEDBACK FOR A CLASS OF NONLINEAR DISCRETE-TIME CONTROL SYSTEMS
}

\author{
M. Bensoubaya A. Ferfera A. Iggidr \\ CONGE Project \\ INRIA Lorraine \& University of Metz (Dept. of Mathematics, URA-CNRS 399) \\ 4, rue Marconi 57070 METZ - FRANCE \\ e.mail: \{bensouba,ferfera,iggidr\}@ilm.loria.fr
}

\begin{abstract}
The goal of this paper is to introduce and to use timevarying control for the stabilization of nonlinear discretetime control systems which can not be stabilized by state feedback.
\end{abstract}

Keywords: stabilization, nonautonomous systems, Lyapunov functions.

\section{Introduction}

The stabilization problem of nonlinear continuous systems has attracted the interest of an increasing number of authors in the last decay (see [8] and references therein). For discrete-time systems there is a few results concerning the stabilization $[1,2,3,9]$. Recently in [1] we have given the following necessary condition for the existence of a continuous stabilizing state feedback :

Theorem 1. Given a discrete-time nonlinear system

$$
x(k+1)=f(x(k), u(k))
$$

where $x \in \mathbb{R}^{n}, u \in \mathbb{R}^{m}$, and $f$ is a continuous function in a neighborhood $A \times U$ of $(0,0)$ satisfying $f(0,0)=0$, a necessary condition for the existence of a continuous feedback $u(x)$, with $u(0)=0$, stabilizing the system (1) is that the image of the map $\gamma: A \times U \rightarrow \mathbb{R}^{n}$ defined by $\gamma(x, u)=f(x, u)-x$ contains some neighborhood of the origin.

In this paper we are interested in the stabilization of nonlinear discrete-time systems of the form

$$
x(k+1)=x(k)+\sum_{i=1}^{m} u_{i}(k) g_{i}(x(k))
$$

where $x=\left(x_{1}, \ldots, x_{n}\right)^{\mathrm{T}} \in \mathbb{R}^{n}, u=\left(u_{1}, \ldots, u_{m}\right)^{\mathrm{T}} \in \mathbb{R}^{m}$, and $g_{i}: \mathbb{R}^{n} \rightarrow \mathbb{R}^{n}, i=1, \ldots, m(m \geq 2)$, are continuous functions on $\mathbb{R}^{n}$. Those systems are not generally stabilizable by continuous state feedback:

Proposition. If $m<n$ then system (2) can not be stabilized by means of continuous state feedback.
Proof: Since $\operatorname{rank}\left\{g_{1}(0), \ldots, g_{m}(0)\right\} \leq m<n$, the map $\gamma: \mathbb{R}^{n} \times \mathbb{R}^{m} \rightarrow \mathbb{R}^{n}$ defined by

$$
\gamma(x, u)=f(x, u)-x=\sum_{i=1}^{m} u_{i}(k) g_{i}(x(k))
$$

is not locally onto. So the necessary condition given by theorem 1 is violated.

As for the continuous systems $[4,6,7]$ this impossibilty to stabilize by state feedback motivates the introduction of time-varying control.

\section{Main result}

In the sequel, we limit ourself to the class of non linear systems of the form (2) with

$$
g_{1}=\frac{\partial}{\partial x_{1}}
$$

For all $x \in \mathbb{R}^{n}$ and all $t \in \mathbb{R}$, set $\tilde{x}=\left(x_{2}, \ldots, x_{n}\right)^{\mathrm{T}}$ and let

$$
V(x, t)=\frac{1}{2}\left[\left(x_{1}+h(\tilde{x}, t)\right)^{2}+\sum_{i=2}^{n} x_{i}^{2}\right]
$$

where $h$ is any $\mathcal{C}^{2}$ function satisfying

$$
\begin{aligned}
h(\tilde{x}, t+2 \pi) & =h(\tilde{x}, t), \quad \forall(\tilde{x}, t) \\
h(0, t) & =0, \quad \forall t
\end{aligned}
$$

A possible choice for $h$ satisfying (5-6) is

$$
h(\tilde{x}, t)=\psi(\tilde{x}) \cos t
$$

where $\psi$ is linear. So $V$ is $2 \pi$-periodic with respect to time $t$, vanishes if and only if $x=0$ and satisfy

$$
\left\{(x, t) \in \mathbb{R}^{n+1} \mid V(x, t) \leq K \text { and }|t| \leq K\right\}
$$

is bounded $\forall K>0$. Besides, define $v: \mathbb{R}^{n+1} \rightarrow \mathbb{R}$ and $f, \tilde{g}_{i}: \mathbb{R}^{n+1} \rightarrow \mathbb{R}^{n+1}(1 \leq i \leq m)$ by

$$
v(x, t)=-\frac{1}{2}\left(x_{1}-h(\tilde{x}, t)\right)-h(\tilde{x}, t+1)
$$




$$
\begin{gathered}
f(x, t)=\left(\begin{array}{c}
x+v(x, t) g_{1}(x) \\
t+1
\end{array}\right)=\left(\begin{array}{c}
x_{1}+v(x, t) \\
\tilde{x} \\
t+1
\end{array}\right) \\
\tilde{g}_{i}(x, t)=\left(\begin{array}{c}
g_{i}(x) \\
0
\end{array}\right)
\end{gathered}
$$

Now we can state and prove the main theorem of this paper.

Theorem 2. Assume that the functions $\tilde{g}_{1}, \ldots, \tilde{g}_{m}$ and $f$ satisfy for any $(x, t) \in \mathbb{R}^{n} \times S^{1}$

$$
\operatorname{rank}\left(\left\{\tilde{g}_{i}\left(f^{k}(x, t)\right), k \in \mathbb{N}, 2 \leq i \leq m\right\}\right)=n-1
$$

Then the origin is a globally uniformly asymptotically stable equilibrium point of the time-varying periodic closed-loop system (2) with

$$
\begin{aligned}
u(x, t)= & \left(\begin{array}{c}
v(x, t) \\
0
\end{array}\right) \\
& -M^{-1}(x, t)(\nabla V(f(x, t)) \tilde{g}(x, t))^{\mathrm{T}}
\end{aligned}
$$

where $M(x, t)=I+\frac{1}{2} g^{\mathrm{T}}(x) \frac{\partial^{2} V}{\partial x^{2}}(f(x, t)) g(x)$.

Proof: The functions $V$ and $v$ from $\mathbb{R}^{n} \times \mathbb{R}$ to $\mathbb{R}$ are both $2 \pi$-periodic with respect to time $t$. So they induce naturally some functions from $\mathbb{R}^{n} \times S^{1}$ to $\mathbb{R}$. Hence, by taking the change of control

$$
\begin{aligned}
& u_{1}=v(x, t)+\tilde{u}_{1} \\
& u_{i}=\tilde{u}_{i}, \quad 2 \leq i \leq m
\end{aligned}
$$

the time-varying closed-loop system can be considered as an autonomous control system on $\mathbb{R}^{n} \times S^{1}$ defined by

$$
\left\{\begin{array}{c}
x(k+1)=x(k)+v(x(k), \theta(k)) g_{1}(x(k)) \\
+\sum_{i=1}^{m} \tilde{u}_{i}(k) g_{i}(x(k)) \\
\theta(k+1)=\theta(k)+1
\end{array}\right.
$$

where $\theta \in S^{1}$. The difference of the Lyapunov function $V$ along the trajectories of system (12) is given by

$$
\Delta V(x, \theta)=V(f(x, \theta)+\tilde{g}(x, \theta) \tilde{u})-V(x, \theta)
$$

where $\tilde{g}(x, \theta)=\left(\tilde{g}_{1}(x, \theta), \ldots, \tilde{g}_{m}(x, \theta)\right.$. If one chooses $h$ such that $V(f(x, \theta)+\tilde{g}(x, \theta) \tilde{u})$ is quadratic on $\tilde{u}$ (of the form (7) for instance), one get

$$
\begin{aligned}
\Delta V(x, \theta)=V(f(x, & \theta))-V(x, \theta) \\
& +\tilde{u}^{\mathrm{T}}(\nabla V(f(x, \theta)) \tilde{g}(x, \theta))^{\mathrm{T}} \\
& +\frac{1}{2} \tilde{u}^{\mathrm{T}} g^{\mathrm{T}}(x) \frac{\partial^{2} V}{\partial x^{2}}(f(x, \theta)) g(x) \tilde{u}
\end{aligned}
$$

Furthermore, since $V$ is positive definite on $\mathbb{R}^{n} \times S^{1}$ then, for any $(x, \theta) \in \mathbb{R}^{n} \times S^{1}$,

$$
N(x, \theta)=g^{\mathrm{T}}(x) \frac{\partial^{2} V}{\partial x^{2}}(f(x, \theta)) g(x) \geq 0
$$

As a matter of fact, if there exists $\left(x_{0}, \theta_{0}\right) \in \mathbb{R}^{n} \times S^{1}$ such that the matrix $N\left(x_{0}, \theta_{0}\right)$ has a negative eigenvalue $\lambda\left(x_{0}, \theta_{0}\right)<0$ associted with an eigenvector $v\left(x_{0}, \theta_{0}\right) \in \mathbb{R}^{m}$, then, for any $\alpha \in \mathbb{R}$, one has from the Taylor expansion formula

$$
\begin{aligned}
V\left(f\left(x_{0}, \theta_{0}\right)+\tilde{g}\left(x_{0}, \theta_{0}\right)\right. & \left.\alpha v\left(x_{0}, \theta_{0}\right)\right)= \\
V\left(f\left(x_{0}, \theta_{0}\right)\right)+\nabla V( & \left.f\left(x_{0}, \theta_{0}\right)\right) \tilde{g}\left(x_{0}, \theta_{0}\right) v\left(x_{0}, \theta_{0}\right) \alpha \\
+ & \frac{1}{2} \lambda\left(x_{0}, \theta_{0}\right)\left\|v\left(x_{0}, \theta_{0}\right)\right\|^{2} \alpha^{2}
\end{aligned}
$$

It turns out that

$$
\lim _{\alpha \rightarrow \infty} V\left(f\left(x_{0}, \theta_{0}\right)+\tilde{g}\left(x_{0}, \theta_{0}\right) \alpha v\left(x_{0}, \theta_{0}\right)\right)=-\infty
$$

that provides a contradiction with the fact that $V$ is positive definite on $\mathbb{R}^{n} \times S^{1}$, and so (13) is established. Hence, the matrix

$$
M(x, \theta)=I+\frac{1}{2} g^{\mathrm{T}}(x) \frac{\partial^{2} V}{\partial x^{2}}(f(x, \theta)) g(x)
$$

is invertible. It follows from (4), (8) and (9) that with the feedback law defined on $\mathbb{R}^{n} \times S^{1}$ by

$$
\tilde{u}(x, \theta)=-M^{-1}(x, \theta)(\nabla V(f(x, \theta)) \tilde{g}(x, \theta))^{\mathrm{T}}
$$

the difference of the Lyapunov function $V$ along the trajectories of the closed-loop system (12-14) becomes

$$
\begin{aligned}
\Delta V(x, \theta) & =V(f(x, \theta))-V(x, \theta)-\tilde{u}^{\mathrm{T}}(x, \theta) \tilde{u}(x, \theta) \\
& =-\frac{3}{8}\left(x_{1}+h(\tilde{x}, \theta)\right)^{2}-\tilde{u}^{\mathrm{T}}(x, \theta) \tilde{u}(x, \theta) \leq 0
\end{aligned}
$$

which implies that $V$ decreases along the solutions of the closed-loop system (12-14) and then the origin is Lyapunov stable. To show that zero is unifomly globally asymptotically stable equilibrium point of the closedloop system $(2-11)$ or that the invariant set $\{0\} \times S^{1}$ is globally attractive, set

$$
\begin{aligned}
E & =\left\{(x, \theta) \in \mathbb{R}^{n} \times S^{1} \mid \Delta V(x, \theta)=0\right\} \\
& =\{(x, \theta) \mid V(f(x, \theta))-V(x, \theta)=0, \tilde{u}(x, \theta)=0\}
\end{aligned}
$$

According to LaSalle's invariance principle all the solutions of the closed-loop system (12-14) tend to $\Omega$ the largest invariant set contained in $E$. In order to prove the global attractivity let us show that $\Omega=\{0\} \times S^{1}$. Using (14) one has

$$
\tilde{u}(x, \theta)=0 \Rightarrow \nabla V(f(x, \theta)) \tilde{g}(x, \theta)=0
$$

Let $(x(k), \theta(k))$ be a solution of the closed-loop system with $(x(0), \theta(0))=(x, \theta) \in \Omega$. Since $\Omega$ is invariant for the closed-loop system we have $(x(k), \theta(k)) \in \Omega$ for all $k \geq 0$. But, $\tilde{u}(x, \theta)$ vanishing on $\Omega$, one has $(x(k), \theta(k))=f^{k}(x, \theta)$ and so, by (15) and (16)

$$
V\left(f^{k+1}(x, \theta)\right)-V\left(f^{k}(x, \theta)\right)=0, \quad \forall k \in \mathbb{N}
$$


and

$$
\nabla V\left(f^{k+1}(x, \theta)\right) \tilde{g}\left(f^{k}(x, \theta)\right)=0, \quad \forall k \in \mathbb{N}
$$

Notice that, by (4),

$$
\nabla V(x, \theta)=\left(\begin{array}{c}
x_{1}+h(\tilde{x}, \theta) \\
\tilde{x}+\left(x_{1}+h(\tilde{x}, \theta)\right) \frac{\partial h}{\partial \tilde{x}}(\tilde{x}, \theta) \\
\left(x_{1}+h(\tilde{x}, \theta)\right) \frac{\partial h}{\partial \theta}(\tilde{x}, \theta)
\end{array}\right)
$$

and, by (8) and (9), for any $k \in \mathbb{N}$

$$
f^{k}(x, \theta)=\left(\begin{array}{c}
\frac{1}{2^{k}}\left(x_{1}+h(\tilde{x}, \theta)\right)-h(\tilde{x}, \theta+k) \\
\tilde{x} \\
\theta+k
\end{array}\right)
$$

so that

$$
\nabla V\left(f^{k}(x, \theta)\right)=\left(\begin{array}{c}
\frac{1}{2^{k}}\left(x_{1}+h(\tilde{x}, \theta)\right) \\
\tilde{x}+\frac{1}{2^{k}}\left(x_{1}+h(\tilde{x}, \theta)\right) \frac{\partial h}{\partial \tilde{x}}(\tilde{x}, \theta+k) \\
\frac{1}{2^{k}}\left(x_{1}+h(\tilde{x}, \theta)\right) \frac{\partial h}{\partial \theta}(\tilde{x}, \theta+k)
\end{array}\right)
$$

Now, a simple computation shows that (17) implies

$$
x_{1}+h(\tilde{x}, \theta)=0
$$

and so

$$
\begin{aligned}
& \nabla V\left(f^{k+1}(x, \theta)\right) \tilde{g}\left(f^{k}(x, \theta)\right)= \\
& \left(\left\langle\left(\begin{array}{l}
0 \\
\tilde{x} \\
0
\end{array}\right), \tilde{g_{1}}\left(f^{k}(x, \theta)\right)\right\rangle, \ldots,\left\langle\left(\begin{array}{l}
0 \\
\tilde{x} \\
0
\end{array}\right), \tilde{g_{m}}\left(f^{k}(x, \theta)\right)\right\rangle\right)
\end{aligned}
$$

It turns out from $(3)$ and $(18)$ that $\left(0, \tilde{x}^{\mathrm{T}}, 0\right)^{\mathrm{T}}$ belongs to

$$
\left(\operatorname{Span}\left(\left\{\tilde{g}_{2}\left(f^{k}(x, \theta)\right), \ldots, \tilde{g}_{m}\left(f^{k}(x, \theta)\right), k \in \mathbb{N}\right\}\right)\right)^{\perp}
$$

Hence, it follows from (10) that $\tilde{x}=0$, and so, by (19), $x_{1}=-h(0, \theta)=0$ which finally implies $x=0$.

Example. Consider the system evolving in $\mathbb{R}^{3}$ defined by

$$
\left\{\begin{array}{l}
x(k+1)=x(k)+u_{1}(k) \\
y(k+1)=y(k)+x(k) u_{2}(k) \\
z(k+1)=z(k)+u_{2}(k)
\end{array}\right.
$$

This system doesn't satisfy the necessary stabilizability condition of theorem 1 . So the best we can do is to design a time-varying feedback stabilizer. The asumptions of theorem 2 are satisfied with the Lyapunov function

$$
V(x, y, z, t)=(x+y \cos (t))^{2}+y^{2}+z^{2}
$$

So, one may easily check that this system can be stabilized thanks to the following periodic time-varying feedback:

$$
\begin{gathered}
u_{1}(x(k), y(k), z(k), k)=-\frac{1}{2}[x(k)-y(k) \cos (k) \\
+2 y(k) \cos (k+1)]+\frac{\varphi_{1}(x(k), y(k), z(k), k)}{4+2 x^{2}(k)+x^{2}(k) \cos ^{2}(k+1)} \\
u_{2}(x(k), y(k), z(k), k)=\frac{\varphi_{2}(x(k), y(k), z(k), k)}{4+2 x^{2}(k)+x^{2}(k) \cos ^{2}(k+1)}
\end{gathered}
$$

with

$$
\begin{gathered}
\varphi_{1}(x, y, z, t)=-2 x-x^{3}-6 y \cos (t+1)-x^{2} y \cos (t+1) \\
+2 x z \cos (t+1)+4 y \cos (t+2)+2 x^{2} y \cos (t+2) \\
\varphi_{2}(x, y, z, t)=-4 x y-4 z-x^{2} \cos (t+1) \\
-3 x y \cos ^{2}(t+1)+2 x y \cos (t+1) \cos (t+2)
\end{gathered}
$$

\section{References}

[1] M. Bensoubaya, A. Ferfera and A. Iggidr. Stabilization of discrete-time nonlinear systems. to appear in Comtes rendus de l'Academie des Sciences, Paris.

[2] C.I. Byrnes, W. Lin Losslessness, Feedback Equivalence, and the global Stabilization of discrete-time nonlinear systems. IEEE aut-control, 39, No 1 1994, p. $83-97$.

[3] C.I. Byrnes, W. Lin et B.K. Ghosh, Stabilization of discrete-time nonlinear systems by smooth state feedback, Systems \& Control Letters, 21, 1993, p. 255-263.

[4] J.M. Coron. Global asymptotic stabilization for controllable systems without drift. Math. of Control, Signals and Systems (1992).

[5] J.P. LaSalle, The stability and control of discret Processes, Springer-Verlag, New York, 1986.

[6] J.B. Pomet. Explicit design of time-varying stabilizing control laws for a class of controllable systems without drift Systems \& Control Letters (1992).

[7] C. Samson. Velocity and torque feedback control a nonholonomic cart. Int. Wrkshop in Adaptive and nonlinear Control. Issues in Robotics Grenoble, Springer, 1990, pp 125-151.

[8] E.D. Sontag. Feedback stabilization of nonlinear systems. In Robust Control of Linear Systems and Nonlinear Control, pages 61-81. Eds Kaashoek M.A, van Schuppen J.H, Ran A.C.M, Birkhauser, 1990.

[9] J. Tsinias. Stabilizability of Discrete-Time Nonlinear Systems IMA J. Math. Control and Inf., (6):135-150, (1989). 